\title{
Etnoecologia de pequenos cetáceos: interações entre a pesca artesanal e golfinhos no norte do estado do Rio de Janeiro, Brasil
}

\author{
Gabrielle Amorim Rosa \\ Camilah Antunes Zappes * \\ Ana Paula Madeira Di Beneditto \\ Universidade Estadual do Norte Fluminense, \\ Avenida Alberto Lamego, 2000, CEP 28013-602, Campos dos Goytacazes - RJ, Brasil \\ * Autor para correspondência \\ camilahaz@yahoo.com.br
}

Submetido em 05/08/2011

Aceito para publicação em 03/04/2012

\section{Resumo}

Estudos no norte do Rio de Janeiro indicam interações entre a pesca artesanal e os cetáceos, mas não existem pesquisas que abordem o conhecimento de pescadores sobre estes animais. O objetivo deste estudo é descrever as interações entre os cetáceos e a pesca através da percepção dos pescadores de Atafona (RJ). Entre fevereiro e março de 2010 foram selecionados 20 pescadores através da técnica "bola-de-neve" e aplicado um questionário etnográfico a fim de obter dados através de relatos da percepção cultural sobre os pequenos cetáceos no norte fluminense. Cada pescador descreveu mais de um golfinho, o que explica o tamanho amostral das respostas $(\mathrm{N}=34)$ maior que o número de entrevistados $(\mathrm{N}=20)$. Pelos relatos dos informantes foram identificadas três espécies e um gênero: Sotalia guianensis ( $\mathrm{N}=15 ; 75 \%)$; Pontoporia blainvillei $(\mathrm{N}=9 ; 45 \%)$; Steno bredanensis $(\mathrm{N}=6 ; 30 \%)$ e Stenella $\mathrm{spp}$. ( $\mathrm{N}=4 ; 20 \%)$. A resposta "colisão com artefatos" (rede de espera) foi a única descrita na questão da ocorrência de acidentes entre a pesca e os golfinhos $(\mathrm{N}=24 ; 71 \%)$ e a rede de espera é o artefato responsável pelo emalhe dos animais. As carcaças dos golfinhos emalhados podem ser descartadas ao mar e/ ou a musculatura e gordura utilizadas como isca na pescaria de elasmobrânquios. As quatro principais espécies reportadas na literatura para a área foram as identificadas pelos pescadores. Para todos os entrevistados o emalhe é causado pelos golfinhos o que prejudica a pesca.

Palavras-chave: Captura acidental; Delphinidae; Etnoecologia; Pescadores artesanais

\section{Abstract}

Ethnoecology of small cetaceans: interactions between an artisanal fishery and dolphins in northern Rio de Janeiro, Brazil. Studies in northern Rio de Janeiro indicate there are interactions between fisheries and cetaceans, but there are no studies that focus on the knowledge fishermen have about these animals. This study describes the interactions between cetaceans and a fishery through the perception of fishermen from Atafona (RJ). Between February and March 2010, 20 fishermen were selected using the "snowball" technique. An ethnographic questionnaire was given to each fisherman. Each participant described more than one species of dolphin, which explains why the sample size of the responses $(\mathrm{N}=34)$ is greater than the number of respondents 
$(\mathrm{N}=20)$. Based on the reports, three species and one genus were identified: Sotalia guianensis $(\mathrm{N}=15 ; 75 \%)$, Pontoporia blainvillei $(\mathrm{N}=9 ; 45 \%)$, Steno bredanensis $(\mathrm{N}=6 ; 30 \%)$ and Stenella $(\mathrm{N}=4 ; 20 \%)$. The answer "collision with artifacts" was the only one given for the question about the occurrence of accidents between the fishery and dolphins $(\mathrm{N}=24 ; 71 \%)$; gillnets are responsible for the entanglement of the animals. The carcasses of dolphins killed by accidental capture are discarded into the sea and/or the muscle and blubber is used as bait to fish for elasmobranchs. The dolphin species identified by the fishermen corresponded to the four main species reported in the literature for the area. All interviewees said that bycatch is caused by dolphins and affects fishing.

Key words: Accidental capture; Artisanal fishermen; Delphinidae; Ethnoecology

\section{Introdução}

O estudo do conhecimento de populações humanas sobre os fenômenos naturais, ou seja, a Etnociência pressupõe que cada povo possui sua forma classificatória e uma relação própria com o meio natural e, neste aspecto, cada comunidade deve ser estudada de forma específica. Esta ciência realiza comparações entre o conhecimento praticado pelas comunidades humanas e o descrito pelo meio acadêmico, utilizando a linguística como base metodológica (ROUÉ, 2000; FARIAS; ALVES, 2007). A Etnoecologia, como ramo da Etnociência, permite a integração entre o saber acadêmico e o saber social na área da ecologia, já que é voltada para o estudo do conhecimento dos grupos humanos, suas práticas e crenças em relação ao ecossistema (DIEGUES, 2000; CAMPOS, 2001).

Os pescadores artesanais pertencem às chamadas comunidades tradicionais, cujo conhecimento ecológico local é transmitido entre parentes ou dos pescadores mais velhos para os mais jovens (COSTA-NETO, 2000; CLAUZET et al., 2005; PETERSON et al., 2008). Estes trabalhadores detêm o conhecimento baseado na vivência diária com as espécies aquáticas na região em que praticam a pesca (DIEGUES, 2000). Desta forma, o conhecimento das comunidades de pesca pode representar uma fonte de informações para as pesquisas relacionadas à conservação de animais marinhos (FISHER; YOUNG, 2007). A pesca artesanal é caracterizada como uma atividade praticada para fins de subsistência e comercialização em que são utilizados barcos de pesca pequenos (até $10 \mathrm{~m}$ de comprimento e capacidade para até oito tripulantes) (SANCHES, 2004; PETERSON et al., 2008).
Interações entre cetáceos e a atividade da pesca artesanal são descritas em águas costeiras brasileiras por vários autores (e.g. SIMÕES-LOPES, 1991; SICILIANO, 1994; OTT et al., 2002; FREITAS NETTO; DI BENEDITTO, 2008), incluindo o litoral do estado do Rio de Janeiro (DI BENEDITTO et al., 1998; DI BENEDITTO, 2003; ZAPPES et al., 2009; 2010).

As relações registradas entre a pesca artesanal e os cetáceos podem ser positivas, como a pesca cooperativa que ocorre principalmente no sul do Brasil (PRYOR et al., 1990; SIMÕES-LOPES, 1998; PETERSON et al., 2008; ZAPPES et al., 2011a), e negativas, como a captura ou emaranhamento acidental em artefatos de pesca, colisões com embarcações, arpoamento e roubo de pescado preso aos artefatos de pesca (OTT et al., 2002; DI BENEDITTO, 2003; FREITAS NETTO; DI BENEDITTO, 2008; ZAPPES et al., 2009).

A principal interação negativa entre a pesca e os cetáceos se refere à mortalidade decorrente do envolvimento acidental em atividades de pesca com rede de espera (SICILIANO, 1994; BERTOZZI; ZERBINI, 2002; DI BENEDITTO, 2003; ZAPPES et al., 2009). A utilização deste artefato causa maior impacto sobre as espécies de pequeno porte e com tamanho populacional reduzido (OTT et al., 2002; DI BENEDITTO, 2003; SECCHI et al., 2003). As principais espécies capturadas durante as operações de pesca artesanal praticadas em águas brasileiras são o boto-cinza (Sotalia guianensis VAN BÉNÉDEN, 1864), a toninha (Pontoporia blainvillei GERVAIS; D'ORBIGNY, 1844) e o golfinho-nariz-de-garrafa (Tursiops truncatus MONTAGU, 1821) (SICILIANO, 1994; OTT et al., 2002; FRUET et al., 2010). Para a região norte do estado do Rio de Janeiro, S. guianensis e P. blainvillei também são os golfinhos com maior 
número de enredamentos acidentais registrados (DI BENEDITTO et al., 1998; DI BENEDITTO, 2003).

Apesar da interação negativa entre a pesca e os pequenos cetáceos ser descrita para o norte do estado do Rio de Janeiro (LODI; CAPISTRANO, 1990; SICILIANO, 1994; DI BENEDITTO et al., 1998; DI BENEDITTO, 2003), não existem estudos que retratem o conhecimento local dos pescadores sobre esses mamíferos e a interação destes com a pesca artesanal para o norte fluminense. Para tanto, se faz necessário entender a linguagem do pescador através da sua percepção, em que a partir do entendimento do conhecimento local sobre os pequenos cetáceos é possível propor estratégias que envolvam a participação da comunidade no manejo das populações locais desses animais (ZAPPES et al., 2009). Neste contexto, o objetivo do presente estudo é identificar e descrever a percepção dos pescadores artesanais do porto de Atafona, costa norte do estado do Rio de Janeiro, em relação aos pequenos cetáceos que ocorrem na região através da morfologia das espécies e da classificação dos animais elaborada pelos pescadores locais; como também as interações positivas e conflitos existentes entre estes animais e a comunidade pesqueira local.

\section{Material e Métodos}

\section{Área de estudo}

Este estudo foi realizado em Atafona, distrito pertencente ao município de São João da Barra (21 ${ }^{\circ} 37^{\prime} \mathrm{S}$ $\left.-41^{\circ} 03^{\prime} \mathrm{O}\right)$, litoral norte do estado do Rio de Janeiro, especificamente no porto de Atafona localizado na margem sul da foz principal do rio Paraíba do Sul (Figura 1).

\section{Pesca artesanal no norte fluminense}

No porto de Atafona a pesca artesanal utiliza artefatos que pertencem à divisão das redes, como

FIGURA 1: Mapa da costa norte do estado do Rio de Janeiro, com localização do porto de Atafona.

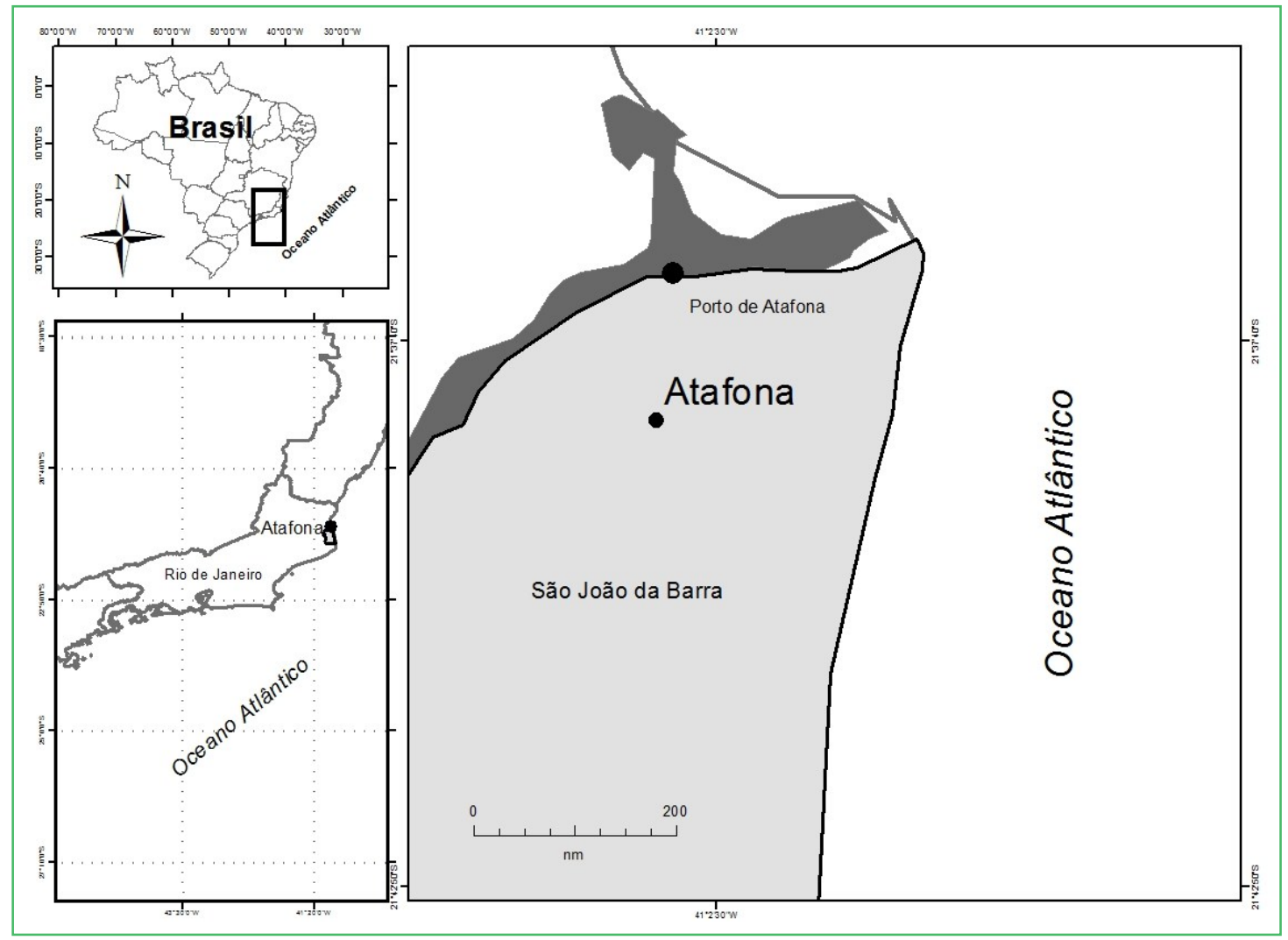


redes de espera e de arrasto, à divisão de linhas, como pargueira, linha de fundo, de superfície e espinhel e à divisão das armadilhas, como o puçá. As embarcações possuem de 9 a $13 \mathrm{~m}$ de comprimento, com capacidade de carga que varia de menos de 1 (uma) a 6 t e tripulação formada por 2 a 5 pescadores (DI BENEDITTO, 2001). Segundo DI BENEDITTO (2003), cerca de 50 barcos sediados no porto de Atafona operam com redes de espera que variam de $1,3-4,9 \mathrm{~km}$ de comprimento, altura entre $1,9-6,0 \mathrm{~m}$ e tamanho da malha entre $100-$ $300 \mathrm{~mm}$. Essas redes de espera são armadas no mar de 1 a 8 vezes por semana e podem ficar até 12 horas na água. O tempo de embarque para a pescaria varia de 2 a 4 semanas/mês ou 2 a 6 dias na semana. Os principais recursos pesqueiros da região são representados pelo camarão sete-barbas (Xiphopenaeus kroyeri, Penaeidae) e por peixes das famílias Balistidae, Carangidae, Carcharhinidae, Coryphaenidae, Pomadasyidae, Pomatomidae, Sciaenidae, Scombridae e Sparidae (DI BENEDITTO et al., 2001a).

\section{Procedimentos}

Entre os meses de fevereiro e março de 2010 foram realizadas 20 entrevistas etnográficas com os pescadores artesanais associados à Colônia de Pescadores Z-2, Atafona. Para cada entrevistado foram explicados os propósitos da pesquisa, sendo solicitada a Anuência Prévia ao presidente da Colônia de Pescadores para realização do estudo. Nesta colônia estão cadastrados 120 pescadores artesanais que atuam somente ao longo da linha de costa do norte do estado e não possuem experiência na pesca industrial. O número de entrevistas $(\mathrm{N}=20)$ permitiu a obtenção de informações consistentes referentes aos pequenos cetáceos e à pesca artesanal praticada no norte fluminense, o que foi considerado suficiente para caracterizar um tamanho amostral ideal como sugerido por Alexiades (1996).

Três critérios de escolha dos entrevistados foram utilizados, conforme indicado a seguir:

1) Para o primeiro contato com o presidente da Colônia de Pescadores Z-2, a pesquisadora (G.A. Rosa) foi acompanhada por uma agente intermediária (S.R. Gomes; presidente da Associação de Moradores de um bairro de Atafona) a fim de adquirir a confiança dos entrevistados. Esta agente apresentou a função de guia e conduziu a pesquisadora pela região (SALDANHA, 2005; SANTOS, 2006).

2) A seleção do primeiro entrevistado foi feita com o auxílio do presidente da Colônia de Pescadores Z-2, já que geralmente é aquele que melhor conhece os demais (SANCHES, 2004).

3) A partir do segundo entrevistado foi utilizada a técnica bola-de-neve, no qual um potencial entrevistado é indicado pelos membros que já responderam ao questionário (BAILEY, 1994). Esta técnica trabalha com cadeias de referência para o recrutamento fazendo uso das relações entre as pessoas. Assim, após cada entrevista foi solicitado ao entrevistado que indicasse outros pescadores que poderiam participar do estudo e o processo se repetiu com novos entrevistados formando uma rede (PANTON, 1990). Estas indicações de novos membros permitiram o surgimento de pontos de inserção variados (GOODMAN, 1961). Através deste método torna-se mais fácil aos pesquisadores identificar novos potenciais informantes para a pesquisa.

Para a seleção dos entrevistados foram estabelecidos os seguintes critérios: (1) ser pescador artesanal, (2) ter a pesca como principal atividade econômica e (3) praticar a pesca artesanal no norte do estado do Rio de Janeiro. Todos os pescadores foram entrevistados individualmente, evitando-se a interferência do relato de um entrevistado sobre o outro, e as entrevistas foram conduzidas de acordo com a disponibilidade de cada um, ou na residência do informante ou no porto, após o desembarque do pescado. A fim de evitar a indução de respostas não foram utilizadas durante o trabalho de campo camisetas ou objetos com indicações conservacionistas.

Foi aplicado durante a entrevista, um questionário padrão pré-estabelecido (KENDALL, 2008) contendo questões semi-estruturadas com perguntas abertas ( $\mathrm{N}=58)$ e fechadas ( $\mathrm{N}=17$ ) (SCHENSUL et al., 1999). A utilização deste tipo de questionário teve como objetivo não estabelecer um roteiro fechado, o que possibilitou o 
surgimento de novas informações durante a entrevista (QUIVY; CAMPENHOUDT, 1992).

As perguntas contidas no questionário eram referentes aos seguintes aspectos: (1) características sócio-econômicas do pescador (idade, residência e tempo de atuação na atividade pesqueira), (2) características da atividade pesqueira (artefatos, embarcações, peixes de interesse comercial) (BEGOSSI, 2001), (3) características das espécies de pequenos cetáceos que ocorrem na região, (4) interações que os entrevistados considerassem negativas (p. ex. colisão com embarcações e/ou artefatos, golfinho ficar preso em artefato e golfinho atrapalhar a atividade de pesca artesanal) e positivas (p. ex. golfinho auxiliar durante a prática pesca) entre esses animais e a pesca artesanal e (5) soluções para possíveis conflitos que os pescadores considerassem existir. Inicialmente, as perguntas se relacionavam aos golfinhos adultos e, posteriormente, aos infantes (filhote recém-nascido e juvenil). Desse modo, foi possível aos entrevistados descrever com clareza cada uma das duas faixas etárias abordadas. Uma prancha ilustrativa contendo fotos de pequenos cetáceos que ocorrem na região foi utilizada como ferramenta do método da etnografia visual para verificar o reconhecimento e diferenciação entre as espécies descritas pelo entrevistado (ALARCON; SCHIAVETTI, 2005; DARBON, 2005; MIRANDA et al., 2007). Esta prancha foi apresentada ao final da entrevista em que foram inseridas as fotos das seguintes espécies: Stenella frontalis, P. blainvillei, Steno bredanensis, Stenella clymene, Delphinus delphis, Tursiops truncatus, S. guianensis e Globicephala macrorhynchus a fim de confirmar se a espécie que o pescador descreveu também era identificada por ele através da fotografia. Foi explicado aos entrevistados que o tamanho dos animais nas fotografias não estava relacionado ao tamanho real dos mesmos.

\section{Análises de dados}

As respostas foram agrupadas em temas (características do pescador, da atividade pesqueira e dos cetáceos; interações entre os animais e a pesca e soluções para os conflitos) como meio de interpretar os relatos (BOGDAN; BIKLEN, 1994). Por meio da aplicação da análise do discurso foi possível analisar o conhecimento local sobre os pequenos cetáceos e a interação entre a pesca artesanal e estes golfinhos (ROCHA; DEUSDARÁ, 2005). Esta análise foi realizada por meio de macro-estruturas em que foi estabelecido um resumo de várias proposições das falas dos pescadores relacionadas às perguntas do questionário que abordaram as interações entre os animais e a pesca e as soluções para os conflitos (VAN DIJK, 1988).

Para confirmar a identificação dos pequenos cetáceos pelos entrevistados foram analisados os relatos que descreveram as características de cada espécie relacionadas à coloração e comprimento corporal, área de ocorrência e comportamento dos animais (e.g. comportamento exibido nas áreas de pesca, frente às embarcações, durante atividades de socialização e alimentação), além da fotografia que o pescador indicava na prancha como sendo a espécie descrita por ele. Estas descrições foram comparadas com os dados de literatura disponíveis para cada espécie (Tabela 1). A fim de responder as questões sobre os conflitos entre a pesca artesanal e os animais foi analisada a diferença entre proporções no uso das carcaças capturadas acidentalmente e o valor de $p$ igual ou menor a 0,05 foi aplicado para identificar se as amostras são significativamente diferentes através do Teste de Diferença entre Proporções (PROGRAMA STATISTICA 8.0). As porcentagens da identificação foram calculadas utilizando-se o número de relatos encontrados para cada espécie sobre o tamanho amostral de 20 entrevistados.

\section{Resultados e Discussão}

\section{Espécies identificadas}

Todos os entrevistados identificaram uma ou mais espécie (s) e um gênero de pequenos cetáceos que ocorrem na região (Tabela 1). Cada entrevistado descreveu mais de um animal, o que explica o tamanho amostral das respostas $(\mathrm{N}=34)$ maior que o número de entrevistados.

Através dos relatos dos entrevistados os golfinhos descritos foram identificados em quatro espécies, em que 
S. guianensis $(\mathrm{N}=15 ; 75 \%)$ foi a mais frequentemente descrita (Tabela 2). Não foi possível identificar através dos relatos quais as espécies do gênero Stenella foram referidas pelos entrevistados. As espécies deste gênero reportadas pela literatura para o estado do Rio de Janeiro apresentam características morfológicas semelhantes e se distribuem em áreas afastadas da linha de costa (DI BENEDITTO et al., 1998; MORENO et al., 2005). Isto pode confundir os pescadores que no momento em que observam a espécie em seu ambiente natural, podem não perceber as características específicas em função da semelhança entre as espécies.

Seis animais descritos não condizem com a literatura científica, pois através das características relatadas (tamanho corporal, coloração, área de ocorrência e comportamentos) os entrevistados demonstraram confundir a espécie T. truncatus (golfinho nariz-de-garrafa) e indivíduos jovens do gênero Stenella. Indivíduos juvenis, das espécies do gênero Stenella, não apresentam manchas pelo corpo como os adultos, e possuem coloração semelhante à T. truncatus, além de se distribuírem entre as isóbatas de 170-4.500m no oceano Atlântico Sul Ocidental (MORENO et al., 2005). Desta forma, podem ser facilmente confundidos uns com os outros na percepção do observador não especialista, como no caso dos pescadores artesanais. Apesar de serem reportadas populações costeiras de T. truncatus em algumas áreas do Brasil (CASTELLO; PINEDO, 1977; PRYOR et al., 1990; SIMÕES-LOPES, 1991; BARBOSA et al., 2008), para o norte do estado do Rio de Janeiro esta espécie apresenta hábito oceânico (DI BENEDITTO et al., 2001c).

No presente estudo, nenhum relato de golfinho com hábito costeiro e com características morfológicas e categorias comportamentais semelhantes à T. truncatus foi identificado como pertencente a esta espécie, já que para a região estes animais apresentam distribuição oceânica.

TABELA 1: Identificação das espécies de pequenos cetáceos através das características descritas pelos pescadores e comparadas com os dados reportados na literatura.

\begin{tabular}{|c|c|c|c|c|c|}
\hline Delphinidae & $\begin{array}{c}\text { Coloração } \\
\text { Corporal } \\
\end{array}$ & $\begin{array}{c}\text { Comprimento } \\
\text { corporal }\end{array}$ & $\begin{array}{c}\text { Área de } \\
\text { ocorrência* }\end{array}$ & Comportamento* & Referências \\
\hline $\begin{array}{l}\text { Sotalia } \\
\text { guianensis }\end{array}$ & $\begin{array}{l}\text { Rosa no ventre, } \\
\text { azulado, cinza claro e } \\
\text { escuro, meio cinzento } \\
\text { no dorso }\end{array}$ & 1,0 a $2,5 \mathrm{~m}$ & Praia e costa & $\begin{array}{l}\text { Se afasta de embarcações, } \\
\text { poucos comportamentos } \\
\text { aéreos, forrageio na praia }\end{array}$ & $\begin{array}{l}\text { Borobia et al., 1991; Jefferson et } \\
\text { al., 1993; Monteiro-Filho, 1995; } \\
\text { Di Beneditto et al., 2001b ; Daura- } \\
\text { Jorge et al., } 2007\end{array}$ \\
\hline $\begin{array}{l}\text { Pontoporia } \\
\text { blainvillei }\end{array}$ & $\begin{array}{l}\text { Amarelado, rosado, } \\
\text { marrom, cinza claro } \\
\text { amarronzado }\end{array}$ & $60 \mathrm{~cm}$ a $1,5 \mathrm{~m}$ & Praia & $\begin{array}{l}\text { Natação, forrageio } \\
\text { próximo à costa, não se } \\
\text { aproxima de embarcações }\end{array}$ & $\begin{array}{l}\text { Jefferson et al., 1993; Di Beneditto } \\
\text { et al., 2001b; Ott et al., 2002; } \\
\text { Danilewicz et al., } 2009\end{array}$ \\
\hline $\begin{array}{l}\text { Steno } \\
\text { bredanensis }\end{array}$ & $\begin{array}{l}\text { Cinza escuro, dorso } \\
\text { escuro, preto }\end{array}$ & 2,0 a $4,0 \mathrm{~m}$ & $\begin{array}{l}\text { Costa e alto } \\
\text { mar }\end{array}$ & $\begin{array}{l}\text { Acompanha embarcações, } \\
\text { deslocamento (natação) } \\
\text { de agregações que podem } \\
\text { variar em grupos de } 10 \text { a } \\
50 \text { indivíduos. }\end{array}$ & $\begin{array}{l}\text { Ritter, 2002; Gannier e West, 2005; } \\
\text { Kuczaj e Yeater, } 2007\end{array}$ \\
\hline Stenella spp. & $\begin{array}{l}\text { Cinza escuro, preto, } \\
\text { pintado }\end{array}$ & $2,2 \mathrm{~m}$ a $3,0 \mathrm{~m}$ & Alto mar & $\begin{array}{l}\text { Natação, avistagem de } \\
\text { grupos grandes com mais } \\
\text { de } 30 \text { indivíduos }\end{array}$ & $\begin{array}{l}\text { Jefferson et al., 1993; Fertl et al., } \\
\text { 2003; Moreno et al., 2005; Adams e } \\
\text { Rosel, } 2006\end{array}$ \\
\hline
\end{tabular}

\footnotetext{
* Na percepção dos entrevistados, a área de ocorrência 'praia' equivale ao local em que banhistas se distribuem (profundidade aproximada de até 4,0m); 'costa' abrange a área após a praia até o alcance da vista (aproximadamente 2.000m da linha de costa); 'alto mar' equivale às águas profundas acima de $500 \mathrm{~m}$ de profundidade, aproximadamente. O critério ‘comportamento' está relacionado aos comportamentos exibidos pelas espécies nas áreas de pesca (período diurno e noturno) frente às embarcações, durante atividades de socialização e alimentação.
} 
TABELA 2: Espécies e gênero de pequenos cetáceos identificados pelos pescadores artesanais de Atafona, norte do estado do Rio de Janeiro $(\mathrm{N}=20)$.

\begin{tabular}{lcc}
\multicolumn{1}{c}{ Espécies } & N & \% \\
\hline Sotalia guianensis & 15 & $75 \%$ \\
Pontoporia blainvillei & 9 & $45 \%$ \\
Steno bredanensis & 6 & $30 \%$ \\
Stenella spp. & 4 & $20 \%$ \\
\hline
\end{tabular}

A espécie Steno bredanensis poderia confundir os pescadores, pois sua coloração, comprimento corporal e categorias comportamentais se apresentam semelhantes à T. truncatus e indivíduos jovens do gênero Stenella. Uma característica marcante de $S$. bredanensis é a junção entre seu rostro e a base do melão, que se apresenta peculiar e distinta das demais espécies de pequenos cetáceos, facilitando sua identificação. Neste estudo, poucos pescadores locais reconheceram $S$. bredanensis. Apesar de existirem registros para áreas costeiras no Brasil (Ott e Danilewicz, 1996; Di Beneditto et al., 1998), estes se apresentam pontuais o que pode indicar que são animais pouco avistados ou que podem se aproximar pouco da costa, o que justifica a descrição por somente alguns dos entrevistados. Mesmo a espécie sendo citada poucas vezes, os pescadores foram enfáticos ao descreverem a característica da 'cabeça' diferenciada do animal bem como os comportamentos, além de indicarem a fotografia correta na prancha ilustrativa cuja 'cabeça' diferenciada (junção entre o rostro e a base do melão) era visualizada.

As espécies de pequenos cetáceos identificadas neste estudo também foram reconhecidas por pescadores artesanais que atuam em práticas de pesca costeiras em outras regiões do Brasil, como na Baía de Babitonga (SC) em que foram identificadas as espécies $P$. blainvillei e S. guianensis (PINHEIRO; CREMER, 2003), em comunidades do sul do estado da Bahia, norte do Espírito Santo e sul dos estados do Rio de Janeiro e São Paulo (ZAPPES et al., 2009) e na comunidade da Costeira da Armação, na APA de Anhatomirim, estado de Santa Catarina (FERREIRA et al., 2006) onde pescadores reconheceram $S$. guianensis e na comunidade de São Sebastião, estado de São Paulo em que pescadores também identificaram $S$. guianensis, $P$. blainvillei, $S$. bredanensis e espécies do gênero Stenella (SOUZA; BEGOSSI, 2007). A prática da pesca artesanal próxima à costa e o hábito costeiro dos animais indica que tanto os pescadores quanto os golfinhos utilizam uma mesma área. Isso permite que os pescadores avistem os animais com maior frequência e com isso os identifiquem com maior facilidade.

\section{Interações com a pesca}

Quando os pescadores foram questionados se existia alguma interferência positiva dos golfinhos sobre a atividade de pesca artesanal não houve nenhum relato, e a interação negativa relacionada ao fato do animal ficar preso em redes de espera foi citado somente por um entrevistado em relação à $P$. blainvillei (5\%).

Os pescadores foram questionados sobre a ocorrência de acidentes entre os animais e a pesca e consideraram "colisão com artefatos", especificamente rede de espera, o único tipo de acidente com cada espécie identificada ( $\mathrm{N}=24 ; 71 \%)$. Pescadores artesanais do estado do Espírito Santo também descreveram o termo 'colisão com artefatos' (FREITAS NETTO; DI BENEDITTO, 2008) já em outros estudos relacionados ao tema estes relatos sobre acidentes não foram registrados (PINHEIRO; CREMER, 2003; FERREIRA et al., 2006; ZAPPES et al., 2009; 2010; 2011b). No entanto, quando os entrevistados foram questionados especificamente sobre a captura acidental de pequenos cetáceos, todos citaram "ficar preso em rede" (animais presos nas redes de pesca), e a rede de espera foi descrita como o único artefato responsável pela captura na região. Este tipo de rede é também citado como o principal artefato responsável pelo emalhamento de pequenos cetáceos por pescadores de comunidades pesqueiras de Ilhéus (BA) (REIS, 2002), ao longo de 36 portos no litoral do Estado do Espírito Santo (FREITAS NETTO; DI BENEDITTO, 2008), em Prado (BA), Nova Viçosa (BA), Baía de Sepetiba (RJ) e Cananeia (SP) (ZAPPES et al., 2009; 2010), no Arquipélago das Cagarras (RJ) (ZAPPES et al., 2011b), na Baía da Babitonga (SC) (PINHEIRO; CREMER, 2003) e na comunidade da Costeira da Armação (SC) (FERREIRA et al., 2006). Capturas acidentais das espécies identificadas pelos entrevistados são reportadas na literatura para a região 
desde a década de 1990 (LODI; CAPISTRANO, 1990; DI BENEDITTO et al., 1998). Em 13 anos de monitoramento regular da captura acidental de cetáceos na região, a rede de espera foi o único artefato registrado como responsável pela mortalidade de pequenos cetáceos (LODI; CAPISTRANO, 1990; DI BENEDITTO et al., 1998; DI BENEDITTO, 2003). A captura acidental por redes de pesca pode diminuir o número de indivíduos nas populações de cetáceos costeiras ao longo do tempo, afetando as populações que possuem número reduzido de indivíduos (OTT et al., 2002; DI BENEDITTO, 2003; ROSSI-SANTOS et al., 2006; DANIELWICZ et al., 2009; 2010). Para o norte do estado do Rio de Janeiro não existem estudos de estimativa populacional para as espécies de pequenos cetáceos, o que dificulta a compreensão sobre a interferência das capturas acidentais sobre a conservação destes animais na região.

Quando questionados especificamente sobre a ocorrência da captura acidental, os entrevistados não identificaram como parte de uma interferência negativa, nem que esta interferência é originada da pesca sobre os golfinhos, mas sim dos animais sobre a pesca. Apenas um entrevistado descreveu o emalhe de P. blainvillei como parte de uma interferência negativa como descrito anteriormente. Esta percepção se relaciona ao fato dos golfinhos danificarem o material de pesca durante a captura, o que causa prejuízo financeiro para o pescador. Desta forma, na percepção de todos os entrevistados $(\mathrm{N}=20)$ são os golfinhos que interferem negativamente sobre a prática pesqueira já que são os responsáveis pela captura acidental, pois não enxergam a rede de espera na água. Situação semelhante é descrita por Zappes et al. (2009) em um estudo etnobiológico sobre $S$. guianensis envolvendo comunidades pesqueiras do sul do estado da Bahia até o sul do estado de São Paulo e por Zappes et al. (2011b) em estudo relacionado às interações entre T. truncatus e as comunidades pesqueiras que atuam no Arquipélago das Cagarras (RJ).

Por outro lado, notou-se durante a realização deste estudo uma contradição na percepção dos pescadores em relação à captura acidental. Ao mesmo tempo em que os golfinhos são apontados como os responsáveis pelos emalhamentos, há também um consenso entre os pescadores que descreveram o emalhe de que as consequências dos conflitos gerados pelas capturas acidentais são mais intensas sobre os animais do que sobre a atividade pesqueira, pois os animais são mortos pelos artefatos de pesca. O prejuízo financeiro pela perda dos artefatos, apesar de relatado, não foi enfatizado pelos entrevistados como importante. Sobre este aspecto, podemos supor que os pescadores podem ter evitado dar importância ao prejuízo financeiro com receio de transparecer aos pesquisadores algum sentimento negativo em relação aos animais. Isso porque sabem que os golfinhos são protegidos por lei e durante as entrevistas faziam questão de relatar seu conhecimento sobre a legislação vigente do tema.

Em relação ao destino dado às carcaças dos animais capturados acidentalmente, os entrevistados relataram descarte ao $\operatorname{mar}(\mathrm{N}=24 ; 65 \%)$ e utilização da musculatura e gordura da carcaça na confecção de iscas para a captura do cação ( $\mathrm{N}=13 ; 35 \%)$ (Figura 2). Não houve diferença significante entre os relatos sobre o destino dado às carcaças dos animais capturados acidentalmente ( $p=0,091)$ e alguns entrevistados descreveram os dois possíveis destinos para essas carcaças (descarte e uso de iscas). Mesmo com a explicação de que este era um estudo acadêmico, percebeu-se ao longo das entrevistas o receio dos pescadores sobre uma possível fiscalização nas embarcações, já que aparentemente todos os entrevistados possuem o conhecimento de que é proibido capturar golfinhos na costa brasileira, pois sem serem questionados, eles mesmos enfatizavam a proibição da captura destes animais.

O descarte e o aproveitamento das carcaças de pequenos cetáceos (uso como isca) capturados acidentalmente por redes de espera também são descritos em outras áreas do litoral do Brasil como na região norte (SICILIANO, 1994), nos estados da Bahia (ZAPPES et al., 2009); Espírito Santo (FREITAS NETTO; DI BENEDITTO, 2008; ZAPPES et al., 2009); Rio de Janeiro (ZAPPES et al., 2009; 2011b) e Santa Catarina (FERREIRA et al., 2006). Segundo os pescadores, as carcaças são descartadas devido ao excesso de gordura presente no corpo do animal que impossibilita o aproveitamento para o consumo, como também o receio da legislação vigente, já que a captura intencional, qualquer forma de molestamento e aproveitamento das 
FIGURA 2: Resposta dos 20 pescadores artesanais em relação ao destino dado às carcaças dos cetáceos capturados acidentalmente no norte do Estado do Rio de Janeiro.

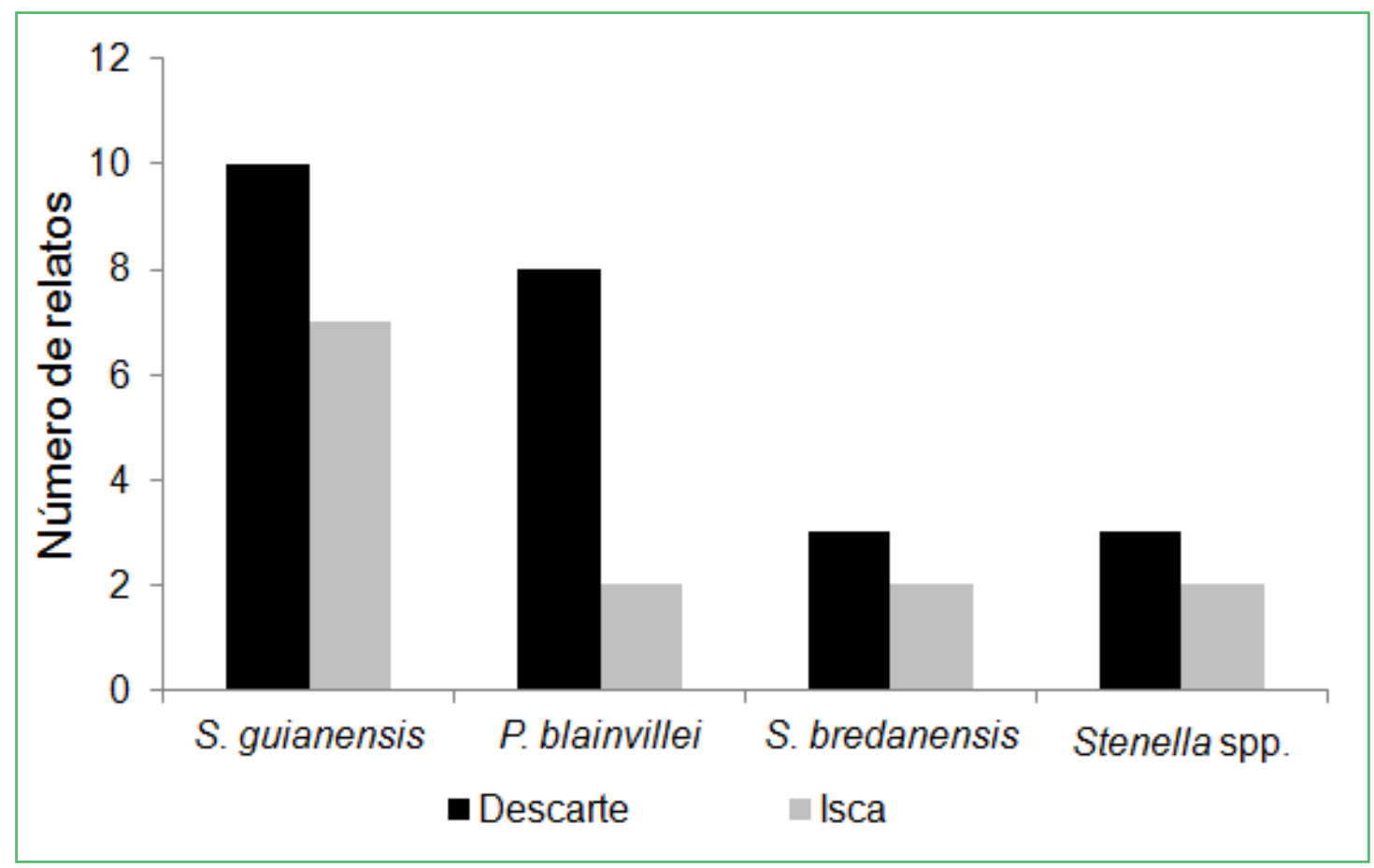

carcaças destes animais é proibida no Brasil (Lei Federal $n^{\circ}$ 7.643/87). Para Zappes et al. (2011b) o descarte é descrito com frequência, pois os pescadores não se sentem à vontade em carregar a carcaça para terra, já que é proibida a manipulação do animal sem permissão. Este sentimento pode mascarar o verdadeiro destino dado às carcaças, uma vez que em muitas situações não é possível aos pesquisadores realizarem observações diretas durante as pescarias (ZAPPES et al., 2009; 2011b).

Siciliano (1994) e Di Beneditto et al. (1998) também descreveram para o porto de Atafona a utilização das carcaças de pequenos cetáceos capturados acidentalmente como isca na captura de cações. Este tipo de uso é uma prática que ocorre na região desde a década de 1960. Atualmente, o principal problema relacionado à conservação das espécies de pequenos cetáceos costeiros no Brasil é a falta de monitoramento regular das capturas acidentais e estimativas dos tamanhos populacionais. Além disso, a insuficiência de informação sobre o esforço de pesca dificulta a estimativa de mortalidade em operações de pesca (OTT et al., 2002). Na área de estudo,
Di Beneditto (2003) sugeriu deslocar as atividades com redes de espera para além de 10 milhas náuticas de distância da linha de costa devido às maiores taxas de capturas acidentais em áreas costeiras, especialmente das espécies $P$. blainvillei e $S$. guianensis.

O entendimento da linguagem local desta comunidade pesqueira auxiliará no monitoramento das atividades de pesca artesanal, especificamente em relação ao uso das redes de espera que são os únicos artefatos que causam a mortalidade acidental dos pequenos cetáceos que se distribuem na região. Com essa aproximação dos pesquisadores sobre a comunidade pesqueira o processo para a conservação pode ser facilitado já que os pescadores podem auxiliar no levantamento de informações concretas sobre o número de capturas acidentais de golfinhos causadas pelas redes de espera no norte fluminense através do uso de diários de bordo além de identificar os locais de sobreposição de área de uso dos animais com a pesca. Esse impacto é mais crítico para as espécies costeiras, S. guianensis e P. blainvillei, e o esforço de estudos etnobiológicos devem ser somados às propostas mitigadoras, como o deslocamento das 
atividades de pesca para além do hábitat preferencial desses golfinhos, de modo que os impactos da pesca artesanal com redes de espera sejam minimizados localmente.

\section{Agradecimentos}

C. A. Zappes agradece à CAPES (Processo 87414) e FAPERJ (E-26/102.798/2011) pela concessão da bolsa de pós-doutorado e A. P. M. Di Beneditto agradece à FA P E R J ( E - $26 / 103.038 / 2008$ ) e CNPq (300241/09-7). Ao presidente da Colônia de Pescadores (Z-12) de Atafona e aos pescadores entrevistados pela colaboração, a Sérgio Moreira pela elaboração do mapa. À Silvana R. Gomes e Camila V. Silva pelo apoio durante a etapa de campo.

\section{Referências}

ADAMS, L. D.; ROSEL, P. E. Population differentiation of the Atlantic spotted dolphin (Stenella frontalis) in the western North Atlantic, including the Gulf of Mexico. Marine Biology, Kiel, v. 148, n. 3, p. 671-681, 2006.

ALARCON, D. T.; SCHIAVETTI, A. O conhecimento dos pescadores artesanais de Itacaré sobre a fauna de vertebrados (não peixes) associados às atividades pesqueiras. Revista de Gerenciamento Costeiro Integrado, Itajaí, v. 4, n. 3, p. 1-4, 2005.

ALEXIADES, M. N. Selected guildelines for ethnobotanical research: a field manual. New York: NYBG, 1996. 306 p.

BAILEY, K. D. Methods of Social Research. New York: The Free Press/Macmillan Publishers, 1994. 588 p.

BARBOSA, M. M. C.; CRUZ, F. S.; LODI, L. Comportamento e organização de grupo do golfinho-flíper, Tursiops truncatus (Cetacea, Delphinidea) no arquipélago das Cagarras, Rio de Janeiro. Revista Brasileira de Zoociências, Juiz de Fora, v. 10, n. 3, p. 209-216, 2008.

BEGOSSI, A. Mapping spots: fishing areas or territories among islanders of the Atlantic Forest (Brazil). Regional Environment Change, Haren, v. 2, n.1, p. 1-12, 2001.

BERTOZZI, C. P.; ZERBINI, A. N. Incidental mortality of franciscana (Pontoporia blainvillei) in the artisanal fishery of Praia Grande, São Paulo state, Brazil. The Latin American Journal of Aquatic Mammals, Rio de Janeiro, v. 1, n. 1, p. 153-160, 2002.

BOGDAN, R. C.; BIKLEN, S. K. Investigação Qualitativa em Educação: Uma Introdução à Teoria e aos Métodos. Porto: Porto Editora LTDA, 1994. 335 p.

BOROBIA, M.; SICILIANO, S.; LODI, L.; HOEK, W. Distribution of the South American dolphin Sotalia Aluviatilis. Canadian Journal of Zoology, Montreal, v. 69, n. 4, p. 1025-1039, 1991.

CASTEllo, H. P.; PINEDO, M. C. Botos na Lagoa dos Patos.
Natureza em Revista, Porto Alegre, v. 12, n. 3, p. 46-49, 1977.

CAMPOS, M. D. Etnociência ou Etnografia de saberes, técnicas e Práticas. In: AMOROZO, M. C. M.; MING, L. C., SILVA, S. P. (Ed.). Métodos de coleta e análises de dados em Etnobiologia, Etnoecologia e disciplinas correlativas. Rio Claro: Universidade Estadual Paulista, 2001. p. 47-92.

CLAUZET, M.; RAMIRES, M.; BARRELlA, W. A. Pesca artesanal e conhecimento local de duas populações caiçaras (enseada do mar virado e barra do una) no litoral de São Paulo, Brasil. Linguagem da Ciência: Multiciência, Campinas, n. 4, p. $1-22,2005$.

COSTA-NETO, E. M. Sustaintable development and tradicional knowledge: a case study in a brazilian artisanal fishermen's community. Sustainable Development, West Sussex, v. 8, n. 2, p. 89-95, 2000.

DANILEWICZ, D.; MORENO, I. B.; OTT, P. H.; TAVARES, M.; AZEVEDO, A. F.; SECCHI, E. R.; ANDRIOLO, A. Abundance estimate for a threatened population of franciscana dolphins in southern coastal Brazil: uncertainties and management implications. Journal of the Marine Biological Association of the United Kingdom, Plymouth, v. 90, n. 8, 1649-1657, 2010.

DANILEWICZ, D.; SECCHI, E. R.; OTT, P. H.; MORENO, I. B.; BASSOI, M.; BORGES-MARTINS, M. Habitat use patterns of franciscana dolphins (Pontoporia blainvillei) off southern Brazil in relation to water depth. Journal of the Marine Biological Association of the United Kingdom, Plymouth, v. 89, n. 5, p. 943949, 2009 .

DARBON, S. O etnólogo e suas imagens. In: SAMAIN, E. (Ed.). O Fotográfico. 2. ed. São Paulo: HUCITEC, 2005. p. 95-105.

DAURA-JORGE, F.; ROSSI-SANTOS, M. R.; WEDEKIN, L. L.; SIMÕES-LOPES, P. C. Behavioral patterns and movement intensity of Sotalia guianensis (P. J. van Bénéden) (Cetacea, Delphinidae) in two different areas on the Brazilian coast. Revista Brasileira de Zoologia, Curitiba v. 24, n. 2, p. 265-270, 2007.

DI BENEDITTO, A. P. M. A pesca artesanal na costa norte do Rio de Janeiro. Bioikos, Campinas, v. 15, n. 2, p. 103-107, 2001.

DI BENEDITTO, A. P. M. Interactions between gillnet fisheries and small cetaceans in northern Rio de Janeiro, Brazil: 2001-2002. The Latin American Journal of Aquatic Mammals, Rio de Janeiro, v. 2, n. 2, p.79-86, 2003.

DI BENEDITTO, A. P. M.; RAMOS, R.; LIMA, N. R. W. Fishing activity in Northern Rio de Janeiro State (Brazil) and its relation with small cetaceans. Brazilian Archives of Biology and Technology, Curitiba, v. 41, n. 3, p. 296-302, 1998.

DI BENEDITTO, A. P. M.; RAMOS, R.; LIMA, N. R. W. Os golfinhos: origem, classificação, captura acidental e hábito alimentar. Porto Alegre: Cinco Continentes Editora, 2001a. 152 p.

DI BENEDITTO, A. P. M.; RAMOS, R.; LIMA, N. R. W. Sightings of Pontoporia blainvillei (Gervais \& D’Orbigny, 1844) and Sotalia guianensis (Gervais, 1853) (Cetacea) in South-eastern Brazil. Brazilian Archives of Biology and Technology, Curitiba, v. 44, n. 3, p. 291-296, 2001 b.

DI BENEDITTO, A. M. P.; RAMOS, R. M. A.; SICILIANO, S.; SANTOS, R. A.; BASTOS, G.; FAGUNDES-NETTO, E. Stomach contents of delphinids from Rio de Janeiro, southeastern Brazil. Aquatic Mammals, Old Mystic, v. 27, n. 1, p. 24-28, 2001c.

DIEGUES, A. C. Etnoconservação da natureza: Enfoques 
alternativos. In: DIEGUES, A. C. (Org.). Etnoconservação: novos rumos para a proteção da natureza nos trópicos. São Paulo: Hucitec LTDA, 2000. p. 1-46.

FARIAS, G. B.; ALVES, Â. G. C. Aspectos históricos e conceituais da etnoornitologia. Biotemas, Florianópolis, v. 20, n.1, p. 91-100, 2007.

FERREIRA, M. C. E.; HANAZAKI, N.; SIMÕES-LOPES, P. C. Os conflitos ambientais e a conservação do boto-cinza na visão da comunidade da Costeira da Armação, na APA de Anhatomirim, Sul do Brasil. Natureza \& Conservação, Curitiba, v. 4, n. 1, p. 64-74, 2006.

FERTL, D.; JEFFERSON, T. A.; MORENO, I. B.; ZERBINI, A. N.; MULLIN, K. D. Distribution of the Clymene dolphin Stenella clymene. Mammal Review, Oxford, v. 33, n. 3-4, p. 253-271, 2003.

FISHER, A.; YOUNG, J. C. Understanding mental constructs of biodiversity: implications for biodiversity management and conservation. Biological Conservation, Orlando, v. 136, n. 2, p. 271-282, 2007.

FREITAS NETTO, R. F.; DI BENEDITTO, A. P. M. Interactions between fisheries and cetaceans in Espírito Santo State coast, southestern Brazil. Revista Brasileira de Zoociências, Juiz de Fora, v. 10, n. 1, p. 55-63, 2008.

FRUET, P. F.; KINAS, P. G.; SILVA, K. G.; DI TULLIO, J. C.; MONTEIRO, D. S.; DALLA ROSA, L.; ESTIMA, S. C.; SECCHI, E. R. Temporal trends in mortality and effects of by-catch on common bottlenose dolphins, Tursiops truncatus, in southern Brazil. Journal of the Marine Biological Association of the United Kingdom, Cambridge, v. 91, p. 1-12.

GANNIER, A.; WEST, K. L. Distribution of the rough-toothed dolphin (Steno bredanensis) around the Windward Islands (French Polynesia). Pacific Science, Honolulu, v. 59, n. 1, p. 17-24, 2005.

GOODMAN, L. Snowball sampling. Annals of Mathematical Statistics, Durham, v. 32, p. 148-170, 1961.

JEFFERSON, T. A.; LEATHERWOOD, S.; WEBBER, M. A. FAO Species Identification Guide: Marine Mammals of the World. Roma: Food and Agriculture Organization, 1993. 320 p.

KENDALL, L. The conduct of qualitative interview: research questions, methodological issues, and researching online. In: COIRO, J.; KNOBEL, M.; LANKSHEA, C.; LEU, D. (Ed.). Handbook of research on new literacies. New York: Lawrence Erlbaum Associates, 2008. p. 133-149.

KUCZAJ, S. A.; YEATER, D. B. Observations of rough-toothed dolphins (Steno bredanensis) off the coast of Utila, Honduras. Journal of the Marine Biological Association of the United Kingdom, Plymouth, v. 87, n. 1, p.141-148, 2007.

LODI, L.; CAPISTRANO, L. Capturas acidentais de pequenos cetáceos no litoral norte do Estado do Rio de Janeiro. Biotemas, Florianópolis, v. 3, n. 1, p. 47-65, 1990.

MIRANDA, T. M.; AMOROZO, M. C. M.; GOVONE, J. S.; DANIELA M. M. The influence of visual stimuli in ethnobotanical data collection using the listing task method. Field Methods, Gainesville, v. 19, n. 1, p. 76-86, 2007.

MONTEIRO-FILHO, E. L. A. Pesca interativa entre o golfinho Sotalia fluviatilis guianensis e a comunidade pesqueira da região de Cananéia. Boletim do Instituto de Pesca, São Paulo, v. 22, n. 2, p. 15-23, 1995.

MORENO, I. B.; ZERBINI, A. N.; DANILEWICZ, D.; SANTOS,
M. C. O.; SIMÕES-LOPES, P. C.; LAILSON-BRITO JR., J.; AZEVEDO, A. F. Distribution and habitat characteristics of dolphins of the genus Stenella (Cetacea: Delphinidae) in the southwest Atlantic Ocean. Marine Ecology Progress Series, Oldendorf/Luhe, v. 300, p. 229-240, 2005.

OTT, P.; DANILEWICZ, D. Southward range extension of Steno bredanensis in the Southwest Atlantic and new records of Stenella coeruleoalba for Brazilian waters. Aquatic Mammals, Old Mystic, v. 22, n. 3, p. 185-189, 1996.

OTT, P.; SECCHI, E.; MORENO, I.; DANILEWICZ, D.; CRESPO, E.; BORDINO, P.; RAMOS, R.; DI BENEDITTO, A. P. M.; BERTOZZI, C.; BASTIDA, R.; KINAS, P. G. Report of the group on fishery interactions. The Latin American Journal of Aquatic Mammals, Rio de Janeiro, v. 1, n. 1, p. 55-64, 2002.

PATTON, M. Q. Qualitative evaluation and research methods. 2. ed. Newbury Park: Sage Publications, 1990. 536 p.

PETERSON, D.; HANAZAKI, N.; SIMÕES-LOPES, P. C. Natural resource appropriation in cooperative artisanal fishing between fishermen and dolphin (Tursiops truncatus) in Laguna, Brazil. Ocean \& Coastal Management, Augustinusga, v. 51, n.6, p. 469$475,2008$.

PINHEIRO, L.; CREMER, M. Etnoecologia e captura acidental de golfinhos (Cetacea: Pontoporidae e Delphinidae) na Baía de Babitonga, Santa Catarina. Desenvolvimento e Meio Ambiente, Curitiba, n. 8, p. 69-75, 2003.

PRYOR, K.; LINDBERGH, J.; LINDBERGH, S.; MILANO, R. A dolphin-human fishing cooperative in Brazil. Marine Mammal Science, Sarasota, v. 6, n. 1, p. 77-82, 1990.

QUIVY, R.; CAMPENHOUDT, L. V. Manual de investigação em ciências sociais. Lisboa: Gradiva, 1992. 275 p.

REIS, M. S. S. O Boto Sotalia fluviatilis (Gervais, 1853) (Cetacea, Delphinidae) no litoral de Ilhéus, Bahia: Comportamento e Interações com as atividades pesqueiras. 2002. 84 f. Dissertação (Mestrado em Desenvolvimento Regional e Meio Ambiente Conservação da Biodiversidade) - Universidade Estadual de Santa Cruz, Ilhéus. 2002.

RITTER, F. Behavioural observations of rough-toothed dolphins (Steno bredanensis) off La Gomera, Canary Islands (1995-2000), with special reference to their interactions with humans. Aquatic Mammals, Old Mystic v. 28, n. 1, p. 46-59, 2002.

ROCHA, D.; DEUSDARÁ, B. Análise de Conteúdo e Análise do Discurso: aproximações e afastamentos na (re)construção de uma trajetória. Alea: Estudos Neolatinos, Rio de Janeiro, v. 7, n. 2, p. 305-322, 2005.

ROSSI-SANTOS, M.; WEDEKIN, L. L.; SOUSA-LIMA, R. $\mathrm{S}$. Distribution and habitat use of small cetaceans off Abrolhos Bank, eastern Brazil. The Latin American Journal of Aquatic Mammals, Rio de Janeiro, v. 5, n. 1, p.23-28, 2006.

ROUÉ, M. Novas Perspectivas em Etnoecologia: "saberes tradicionais" e gestão dos recursos naturais. In: DIEGUES, A. C. (Org.). Etnoconservação novos rumos para a proteção da natureza nos trópicos. 2 ed. São Paulo: NUPAUB-USP, 2000. p. 67-80.

SALDANHA, I. R. R. Espaços, recursos e conhecimento tradicional dos pescadores de manjuba (Anchoviella lepidentostole) em Iguape/SP. 2005. 179 f. Dissertação (Mestrado em Ciência Ambiental) - Universidade de São Paulo, São Paulo. 
2005.

SANCHES, R. A. Caiçaras e a Estação ecológica de Juréia-Itatins. Histórico de ocupação no contexto político, econômico, social e ambiental do Vale do Ribeira. In: MARQUES, O. A. V.; DULEBA, W. (Org.). Estação Ecológica Juréia-Itatins: Ambiente Físico, Flora e Fauna. Ribeirão Preto: Holos Editora, 2004. p. 349-359.

SANTOS, L. M. K. Dinâmica da pesca artesanal em duas comunidades ribeirinhas tradicionais do rio Cuiabá: uma abordagem ecológica. 2006. 87 f. Dissertação (Mestrado em Ecologia e Conservação da Biodiversidade) - Universidade Federal de Mato Grosso, Cuiabá. 2006.

SCHENSUL, S. L.; SCHENSUL, J. J.; LECOMPTE, M. D. Essential Ethnographic Methods: Observations, Interviews and Questionnaires. Walnut Creek: Altamira Press, 1999. 344 p.

SECCHI, E. R.; OTT, P. H.; DANILEWICZ, D. Applying the phylogeographic concept to identify franciscana dolphin stocks: implications to meet management objectives. Journal of Cetacean Research and Management, Cambridge, v. 5, n. 1, p. 61-68, 2003.

SICILIANO, S. Review of small cetaceans and fishery interactions in coastal waters in Brazil. Report of the International Whaling Commission, Cambridge, v. 15, n. 1, p. 241-250, 1994.

SIMÕES-LOPES, P. C. Interactions of coastal populations of Tursiops truncatus (Cetacea, Delphindae) with the mullet artisanal fisheries in Southern Brazil. Biotemas, Florianópolis, v. 4, n. 2, p. 82-95, 1991.

SIMÕES-LOPES, P. C. Intraspecific agonistic behavior of Tursiops truncatus (Cetacea, Delphindae) during dolphin-human cooperative fishing in southern Brazil. Biotemas, Florianópolis, v. 11, n. 2, p.
165-171, 1998.

SOUZA, S. P.; BEGOSSI, A. Whales, dolphins or fishes? The ethnotaxonomy of cetaceans in São Sebastião, Brazil. Journal of Ethnobiology and Ethnomedicine, London, v. 3, n. 9, 2007. http:// www.ethnobiomed.com/content/3/1/9.

VAN DJIK, T. A. News as discourse. Hillsdale: Lawrence Erlbaum Associates, 1988. $197 \mathrm{p}$.

ZAPPES, C. A.; ANDRIOLO, A.; OLIVEIRA, F.; MONTEIROFILHO, E. L. A. Potential conflicts between fishermen and Sotalia guianensis (van Bénéden, 1864) (Cetacea, Delphinidae) in Brazil. Sitientibus Série Ciências Biológicas, Feira de Santana, v. 9, n. 4, p. 208-214, 2009.

ZAPPES, C. A.; ANDRIOLO, A.; SIMÕES-LOPES, P. C.; DI BENEDITTO, A. P. M. 'Human-dolphin (Tursiops truncatus Montagu, 1821) cooperative fishery' and its influence on cast net fishing activities in Barra de Imbé/Tramandaí, Southern Brazil. Ocean \& Coastal Management, Augustinusga, v. 54, n. 5, p. 427 432, 2011a.

ZAPPES, C. A.; GATTS, C. E. N.; LODI, L.; ANDRIOLO, A.; DI BENEDITTO, A. P. M. Interações entre o golfinho-nariz-degarrafa (Tursiops truncatus) e apesca artesanal no Arquipélago das Cagarras e áreas adjacentes, Rio de Janeiro, Brasil. Sitientibus Série Ciências Biológicas, Feira de Santana, v. 11, n. 1, p. 24-30, $2011 b$.

ZAPPES, C. A.; NERY, M. F.; ANDRIOLO, A.; SIMÃO, S. M. Ethnobiology and Photo-identification: identifying anthropic impacts on boto-cinza dolphin Sotalia guianensis in Sepetiba Bay, Brazil. Revista Brasileira de Biociências, Porto Alegre, v. 8, n. 2, p. 221-224, 2010. 